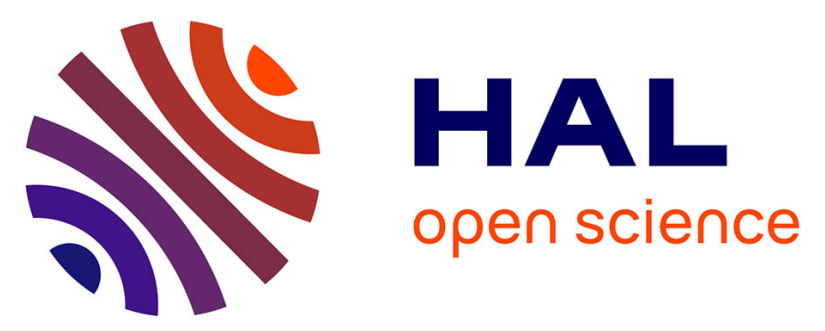

\title{
Automatic segmentation of high pressure frozen and freeze-substituted mouse retina nuclei from FIB-SEM tomograms
}

Thai V Hoang, Caroline Kizilyaprak, Danièle Spehner, Bruno M Humbel, Patrick Schultz

\section{To cite this version:}

Thai V Hoang, Caroline Kizilyaprak, Danièle Spehner, Bruno M Humbel, Patrick Schultz. Automatic segmentation of high pressure frozen and freeze-substituted mouse retina nuclei from FIB-SEM tomograms. Journal of Structural Biology, 2017, 197 (2), pp.123-134. 10.1016/j.jsb.2016.10.005 . hal-03535628

\section{HAL Id: hal-03535628 \\ https://hal.science/hal-03535628}

Submitted on 19 Jan 2022

HAL is a multi-disciplinary open access archive for the deposit and dissemination of scientific research documents, whether they are published or not. The documents may come from teaching and research institutions in France or abroad, or from public or private research centers.
L'archive ouverte pluridisciplinaire HAL, est destinée au dépôt et à la diffusion de documents scientifiques de niveau recherche, publiés ou non, émanant des établissements d'enseignement et de recherche français ou étrangers, des laboratoires publics ou privés. 


\title{
Automatic segmentation of high pressure frozen and freeze-substituted mouse retina nuclei from FIB-SEM tomograms
}

\author{
Thai V. Hoang ${ }^{\text {a,b,c,d }}$, Caroline Kizilyaprak ${ }^{\mathrm{e}}$, Danièle Spehner ${ }^{\mathrm{a}, \mathrm{b}, \mathrm{c}, \mathrm{d}}$, Bruno M. Humbel ${ }^{\mathrm{e}}$, Patrick Schultz ${ }^{\mathrm{a}, \mathrm{b}, \mathrm{c}, \mathrm{d}, *}$ \\ a IGBMC (Institut de Génétique et de Biologie Moléculaire et Cellulaire), 1, rue Laurent Fries, BP 10142, 67404 Illkirch, France \\ ${ }^{\mathrm{b}}$ Inserm, U964, Illkirch, France \\ ${ }^{\mathrm{C}}$ CNRS, UMR7104, Illkirch, France \\ dUniversité de Strasbourg, Strasbourg, France \\ ${ }^{\mathrm{e}}$ University of Lausanne, Electron Microscopy Facility, 1015 Lausanne, Switzerland
}

\section{A R T I C L E I N F O}

\section{Article history:}

Received 4 February 2016

Received in revised form 3 October 2016

Accepted 6 October 2016

Available online $\mathrm{xxxx}$

\section{Keywords:}

FIB/SEM tomography

Segmentation

Nucleus

Heterochromatin

\begin{abstract}
A B S T R A C T
Focused Ion Beam milling combined with Scanning Electron Microscopy is a powerful tool to determine the 3-D organization of whole cells and tissue at an isotropic resolution of 3-5 nm. This opens the possibility to quantify several cellular parameters and to provide detailed phenotypic information in normal or disease states. Here we describe Biocomputing methods to extract in an automated way characteristic features of mouse rod photoreceptor nuclei such as the shape and the volume of the nucleus; the proportion of heterochromatin; the number, density and distribution of nuclear pore complexes (NPC). Values obtained on five nuclei show that the number of NPC $(348 \pm 8)$ is the most conserved feature. Nuclei in higher eukaryotes show large variations in size and rod nuclei are amongst the smallest reported $\left(32 \pm 3 \mu \mathrm{m}^{3}\right.$ ). Despite large species- and cell-type-specific variations in size, the density of NPC (about $\left.15 / \mu \mathrm{m}^{2}\right)$ is highly conserved.
\end{abstract}

(c) 2016 Elsevier Inc. All rights reserved.

\section{Introduction}

The internal organization of cells reflects the expressed part of their genome, determines their specialized role in different tissues, and notifies about their physiological or pathological state. Since the first electron micrograph of cells using transmission electron microscopy (TEM) in 1945 (Porter et al., 1945), electron microscopy (EM) methods have continually improved our appreciation of complex levels of cellular organization. The high spatial resolution of electron microscopes produces a wealth of information about the structure, distribution and interactions between cellular organelles. Early electron microscopy observations of cellular structures were essentially two-dimensional (2-D) and resulted in mostly descriptive information. Stereological methods were used to predict or estimate the size and distribution of cellular structures by extrapolating from 2-D measurements to the whole cellular volume (Weibel et al., 1966). To circumvent limitations due to the intricate shape of cellular structures which prevents straightforward extrapolation, new methods were developed to retrieve 3-D information from cellular samples. By sequentially

\footnotetext{
* Corresponding author at: IGBMC (Institut de Génétique et de Biologie Moléculaire et Cellulaire), 1, rue Laurent Fries, BP10142, 67404 Illkirch, France.

E-mail address: patrick.schultz@igbmc.fr (P. Schultz).
}

tilting the specimen relative to the electron beam, TEM tomography produces a 3-D model of a cell section (Hoppe and Grill, 1977). This powerful method is however limited to $500 \mathrm{~nm}$ in the Z-depth by section thickness and the reconstruction of an entire eukaryotic cell can be very challenging (Noske et al., 2008). Serial thin sections and TEM imaging can solve the problem of the limited Z-depth but this technique is very labor intense and needs highly skilled experimenters (Porter and Blum, 1953; White et al., 1986; Bumbarger et al., 2013). The technological challenge for rapid 3-D imaging of large mammalian cells at nanometric scale was first met with the introduction of block face imaging with a scanning electron microscope (SEM). In this case, the block face of an embedded sample is imaged with sufficient resolution and depth discrimination using the back scattered electron (BSE) detectors. To obtain 3-D data, the block face needs to be repeatedly imaged, with the top slice removed between image acquisitions. One possibility is to remove the top slice with an ultramicrotome in the chamber of a SEM (serial block face, SFB-SEM). This method was introduced 30 years ago by Leighton who constructed a microtome for cutting sections inside the microscope chamber (Leighton, 1981). More recently, Denk and Horstmann showed that with a custom-designed microtome in a SEM, 3-D ultrastructural data can be obtained at the resolution and with a volume sufficient to follow local neuronal circuits (Denk and Horstmann, 2004). 
Currently, the Z-resolution of SBF-SEM of about $25 \mathrm{~nm}$ is limited by the minimal section thickness that can be cut with a diamondknife (Friedrich et al., 2013; Kasthuri et al., 2007; Tapia et al., 2012). The removal of top slices in smaller increments can also be performed by focused gallium ion beam (FIB) milling (Heymann et al., 2006). Recently, the limits of FIB-SEM tomography were pushed to create high-resolution $5 \times 5 \times 5 \mathrm{~nm}$ isotropic voxel acquisition on $40 \times 40 \mu \mathrm{m}$ large image areas and over a depth of several tenths of micrometers (Narayan et al., 2014; Wei et al., 2012). The combination of focused ion beam (FIB) milling and block-face SEM imaging (FIB-SEM tomography) has become a powerful tool for 3-D investigation of cellular ultrastructure and constitutes currently one of the most fast-growing methods to obtain undistorted volume information (Bosch et al., 2015; Cretoiu et al., 2015; De Winter et al., 2009; Hekking et al., 2009; Heymann et al., 2009; Knott et al., 2008; Merchán-Pérez et al., 2009). This new imaging technology opens the possibility to measure morphological parameters on discrete ultrastructural feature. Along with these large 3-D image stacks come many computational challenges to achieve automated 3-D object recognition, annotation, data integration, representation and exploitation. Segmentation is central to these tasks and corresponds to the process of partitioning the 3-D images into multiple structurally defined regions to produce a more meaningful representation suitable for quantitative analysis. This process can be used to delineate the boundaries between cellular compartments or between organelles and cytosol so that quantitative information such as the size, distribution, surface morphology can be extracted from these delineated regions.

Many segmentation techniques have been proposed to target nature scene and medical images. These techniques usually group nearby pixels into regions by optimizing a target function that prefers pixels in the same group to have similar intensity levels and at the same time expects a small number of groups (Arbelaez et al., 2011; Van den Bergh et al., 2014). In spite of many successful applications, these techniques are not suitable for electron microscopy images because of their low signal-to-noise ratio, low contrast and complex image contents. For example, in the presence of noise the performance of watershed transform-based methods (Couprie et al., 2011) decreases rapidly since noise changes the topological landscape of the intensity function representing the image contents and thus breaks biologically-meaningful regions into many small fragments. Recently, some research groups have developed algorithms and software tools that can aid the segmentation of electron microscopy images automatically (NunezIglesias et al., 2014; Schindelin et al., 2012). For boundary segmentation, membrane signals are usually enhanced by filtering and then traced by thresholding (Martinez-Sanchez et al., 2014). For region segmentation, a superpixel-based method is often used to over-segment the image and then a split-and-merge strategy is used for region grouping (Jones et al., 2015). A classifier trained with human annotated data can also be used for direct pixel classification (Sommer et al., 2011). These methods were mostly developed for segmentation of brain tissue images with $25 \mathrm{~nm}$ resolution in the Z-direction. However, most of the developed segmentation algorithms work only with 2-D images and much human effort is still required to correct the automated segmentation results.

Here we have developed algorithms and software tools in a high-throughput computational workflow covering image preprocessing, semi-automatic segmentation of cell nuclei, and extraction of quantitative data from 3-D FIB-SEM images of murine rod photoreceptor cell nuclei. How our genetic information is organized in the cell nucleus and how this organization affects nuclear function are major questions in the chromatin field which remain largely unanswered. In eukaryotes, the genetic information is packaged through a hierarchy of folding events. At the first level DNA is wrapped around an histone octamer to form the nucleosome core particle whose atomic structure is defined (Luger et al., 1997). The fundamental repeated element of chromatin, the nucleosome, is composed of the core particle flanked by linker DNA which connects successive nucleosomes and variable amounts of linker histone H1. Purified chromatin appears as an extended 11-nm fiber formed by a linear beads-on-a-string nucleosomal array that compacts into $30 \mathrm{~nm}$ fibers in physiological ionic strength and in the presence of histone H1 (Oudet et al., 1975; Thoma et al., 1979). Direct electron microscopy imaging of nuclear sections described highly compact electron dense heterochromatin (HC) compartments and more extended euchromatin (EC) territories but has provided little information on chromatin organization beyond the nucleosomal level and in particular has not confirmed the $30-\mathrm{nm}$ fibers as the fundamental in vivo secondary structure of chromatin (Bouchet-Marquis et al., 2005; Horowitz-Scherer and Woodcock, 2005). However, recent publications provide evidence that $30 \mathrm{~nm}$ chromatin fibers may existence in highly condensed chromatin states (Kizilyaprak et al., 2010). The chromatin organization in intact nuclei is thus still a matter of debate.

The tools developed here extend previous quantification efforts of nuclear structures (Rouquette et al., 2009) and assign automatically the extent of the nuclear envelope and measure the size of the nuclei as well as its elongation. The nuclear envelope is perforated by nuclear pores that regulate the exchanges between the nucleus and the cytoplasm. The number and distribution of the pores are determined automatically without using correlation methods. Finally the highly condensed heterochromatin compartment is identified automatically thus opening the possibility to model the rheology of the nucleus within its numerous channels or the diffusion of transcription factors within the nuclei.

\section{Results}

\subsection{Data collection}

To address the quantification of ultrastructural features and particularly the very sensitive nuclei considerations have to be given concerning sample preparation in order to image cells in their closest to native state. In this study, we used high-pressure freezing (HPF) and freeze-substitution (FS) to prepare 4-days old mice retina samples. High-pressure freezing is a method of choice to obtain optimal structural preservation of bulk biological samples with minimal structural rearrangements (Moor et al., 1980). During freeze substitution cryo-immobilized samples were dehydrated by exchange of ice against an organic solvent at low temperature $\left(-90^{\circ} \mathrm{C}\right.$ ) and then embedded in resin (Humbel et al. 1983; Humbel and Schwarz, 1989). High pressure-frozen and freeze-substituted mouse rod photoreceptor nuclei were observed by FIB-SEM tomography and a 3-D images stack was recorded with resolutions of $3.92 \mathrm{~nm}, 3.92 \mathrm{~nm}, 5.00 \mathrm{~nm}$ in the $\mathrm{X}, \mathrm{Y}$, and $\mathrm{Z}$ directions, respectively. The stack contained five complete nuclei that were further analyzed to extract quantitative morphological information (Fig. $1 \mathrm{~A}$ and $7 \mathrm{~A}$ ).

\subsection{Image pretreatment}

Successive images were aligned and their grey-level intensity was normalized. After preprocessing, the images still showed density variations that are not related to the structure of the sample but are detrimental for subsequent image segmentation. A global effect is observed as a continuous intensity variation over the whole imaged area best evidenced in false colors (Fig. 1B). This defect might arise from the SEM optics or variations in secondary 

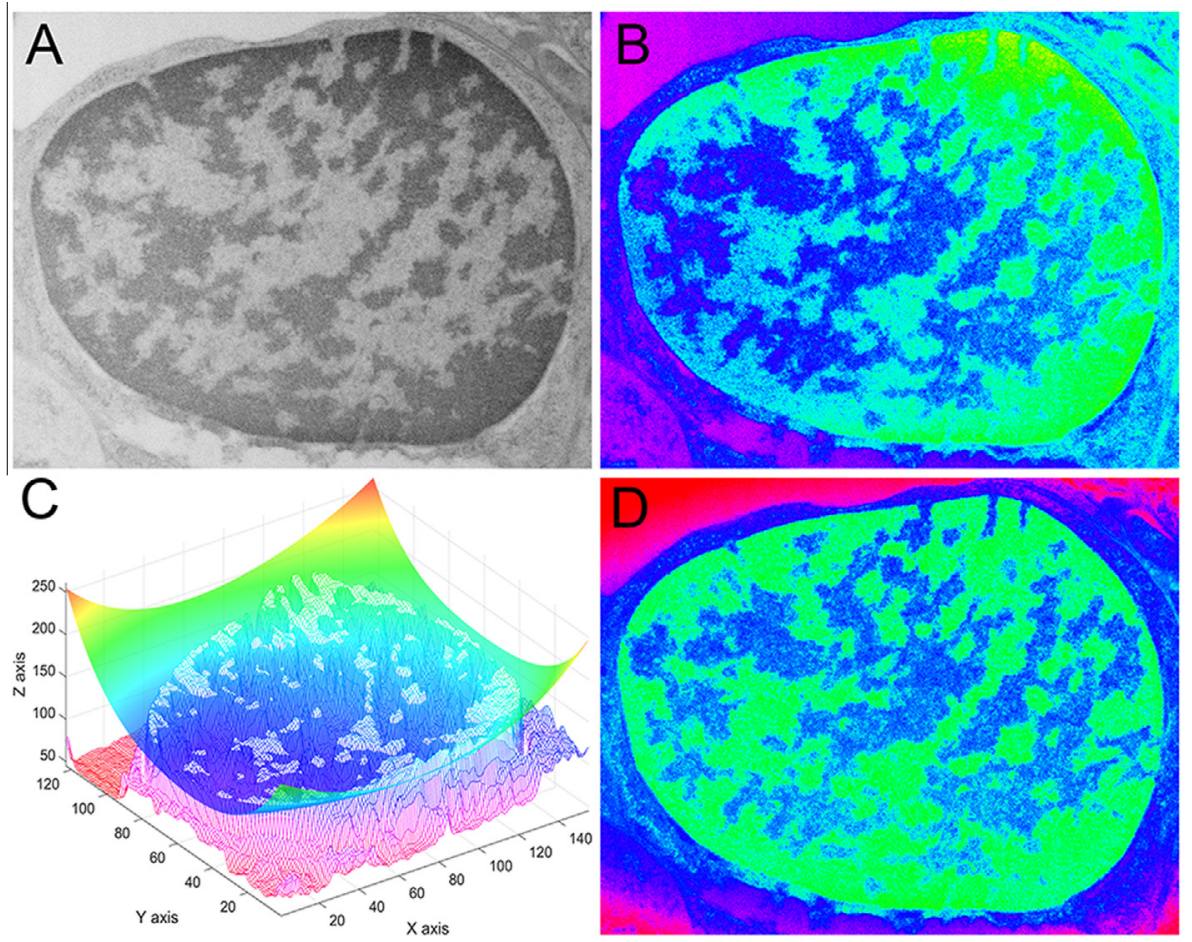

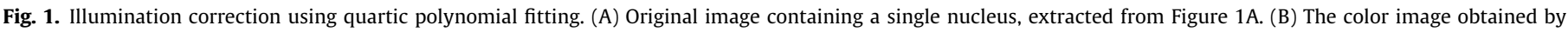

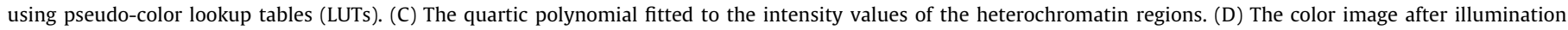
correction.

electron collection efficiency. A second, more subtle intensity variation is restricted to each individual nucleus and is evidenced by the fact that the intensity of the heterochromatin regions slightly declines from the nuclear envelope to its center. This effect is likely to originate from a non-uniform staining of the chromatin during sample preparation but it cannot be excluded that a gradual change in chromatin compaction level gives rise to such an effect (Récamier et al., 2014).

To correct for these effects simultaneously and since reference images are not available, a single-image retrospective methods has been implemented (2) to correct the intensity variations of each nucleus individually based on polynomial surface fitting (Russ, 2011). Polynomials are known to be a good model to estimate gradual variations in brightness. For the nucleus shown in Fig. 1A, the illumination defect is enhanced by false colors (Fig. 1B). The estimated polynomial function is plotted in Fig. $1 \mathrm{C}$ and the corrected image is shown in Fig. 1D. The intensity values of the heterochromatin regions become more homogeneous after correction of systematic intensity variations (compare Fig. 1B and D), thus facilitating their segmentation based on pixel intensity values.

\subsection{Quantification of heterochromatin domains}

The contrasting agents used for the preparation of the retina samples show a strong selectivity for nucleic acids and therefore the image intensity in the nuclei is to the first approximation related to the DNA content. Nuclear domains composed of dark pixels represent highly condensed heterochromatin regions while pixels of higher intensity represent euchromatin corresponding to less condensed chromatin or nucleoplasm that contains little nucleic acids (Fig. 2A). Segmentation of the heterochromatin domains will therefore be based on intensity thresholding. Due to noise in the SEM images and staining heterogeneities, the highly condensed heterochromatin domains cannot be segmented properly on the raw images. Thus, we need to filter the noisy original SEM images while preserving the sharp boundaries and the roughness of the heterochromatin edges. The coherenceenhancing diffusion (CED) algorithm (Weickert, 1999) was employed for this purpose since it has been successfully used to denoise tomography images (Frangakis and Hegerl, 2001). The CED filtered image (Fig. 2B) preserves the sharp heterochromatin boundaries found in the original image.

However, the nuclear envelope in the raw SEM and CED filtered images appears as rough contour, which hinders its segmentation and representation. The block matching 3-D filtering (BM3D) algorithm was used to smoothen the nuclear envelope. The BM3D filtered image (Fig. 2C) has a smooth nuclear envelope but also a drastically reduced roughness of the heterochromatin boundaries, which is not suitable for chromatin segmentation. The pixel intensity distribution after illumination correction and filtering was then used to partition the nucleus into multiple segments (i.e. sets of pixels) in order to delineate the boundaries between heterochromatin and euchromatin regions (Fig. 2D). Since these boundaries are not smooth and are best described by fractal models (Woodcock and Ghosh, 2010), we choose to use histogram-based methods, instead of contour-based ones (Chan et al., 2001), in order to preserve the roughness of these boundaries. The histogram of the pixel intensities clearly shows a bimodal distribution that can be modeled by a combination of two Gaussian distributions (Fig. 2E). The peak of dark pixels with intensity $\mathrm{T}_{1}=24$ corresponds to heterochromatin while the peak of bright pixels centered at $\mathrm{T}_{2}=52$ represents the euchromatin domain. Intermediate pixel intensities (e.g. between 24 and 52) corresponding to discrete levels of DNA compaction could not be resolved in these images. Simply applying a threshold at an intensity value corresponding to the intersection of the two Gaussians (intensity value $T_{3}$ ) produces a noisy segmented image and wrongly assigned pixels appear both in the heterochromatin and euchromatin regions. 

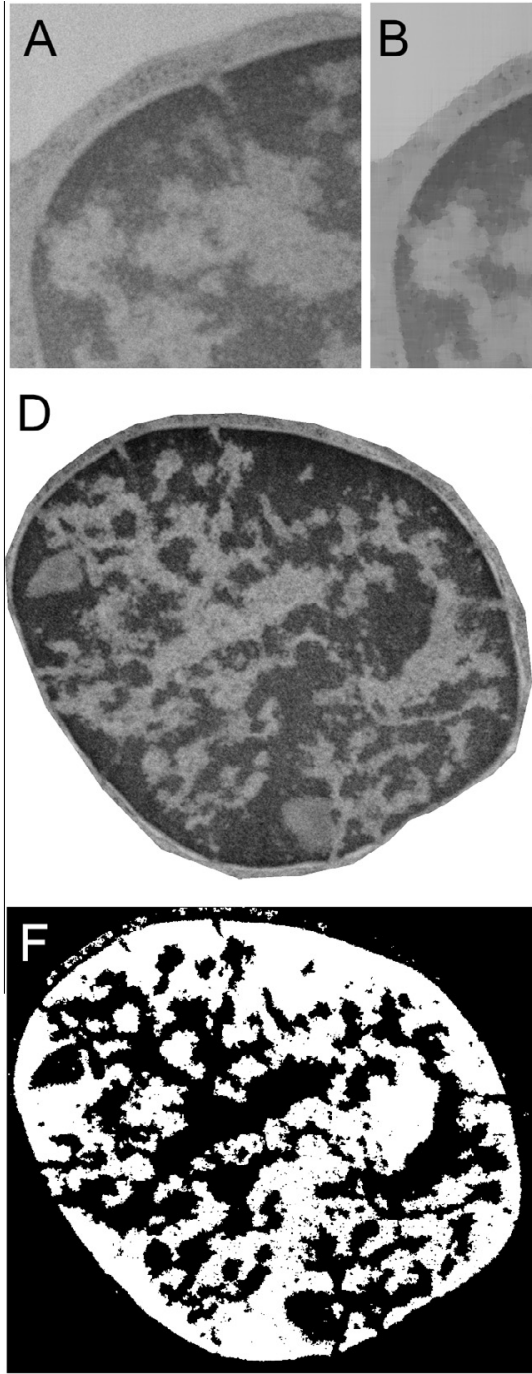

$E$
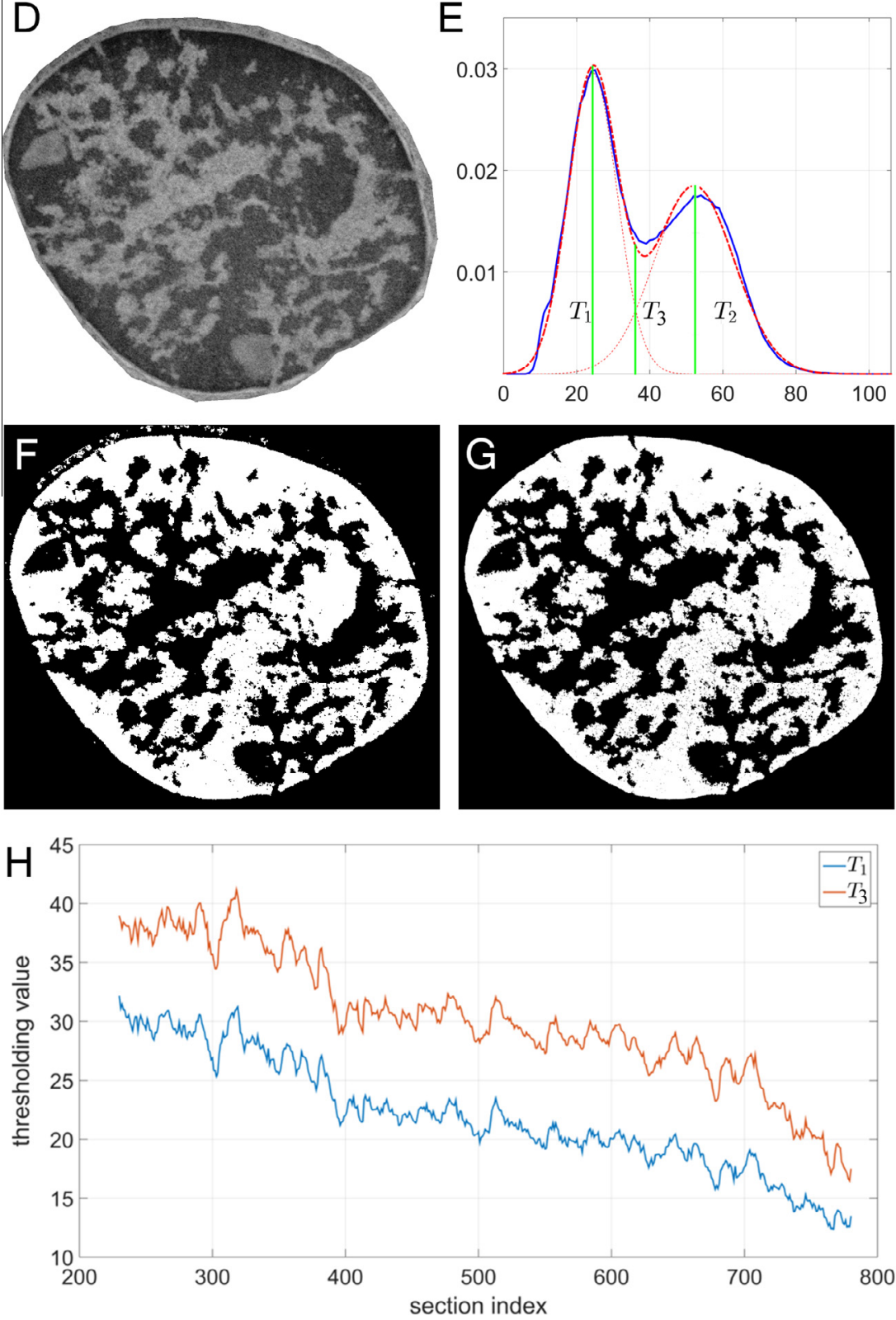

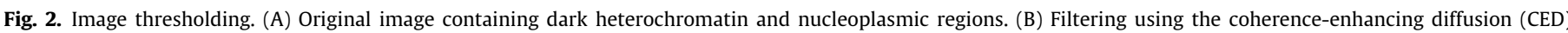

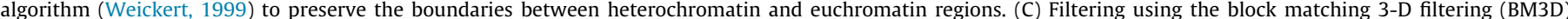

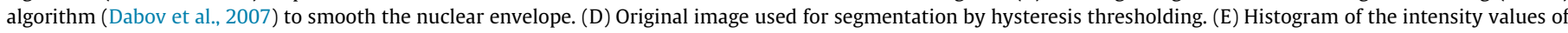

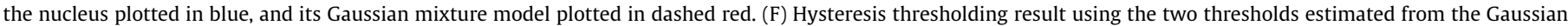

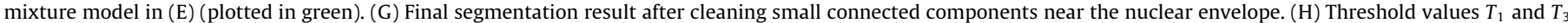

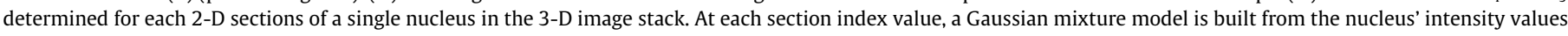
in the corresponding SEM image in order to determine $T_{1}$ and $T_{3}$ at that section index.

We therefore used a connectivity criteria to include pixels with a value between $T_{1}$ and $T_{3}$ only when they are in contact with darker pixels. The hysteresis thresholding technique (Medina-Carnicer et al., 2009) was therefore used in which the image is first 
thresholded to keep pixels with intensity I comprised between $\mathrm{T}_{1}$ and $\mathrm{T}_{3}$ are included only if connected to the dark pixels (Fig. $2 \mathrm{~F}$ ). The remaining noise outside the nuclear envelope can be removed by using the eroding morphological operations (Gonzalez and Woods, 2007) to obtain the final segmentation result (Fig. 2G), in which the nuclear envelop and the heterochromatin boundaries are well delineated.

The set of threshold values $T_{1}$ and $T_{2}$ were determined for each 2-D section of a single nucleus and were not found constant, but rather gradually to decrease with the section index (i.e. as more sections are milled away from the sample). This result suggests that, in order to segment a single nucleus, we should threshold individually each 2-D SEM images rather than use a single set of value for the whole image stack. Moreover we found that this
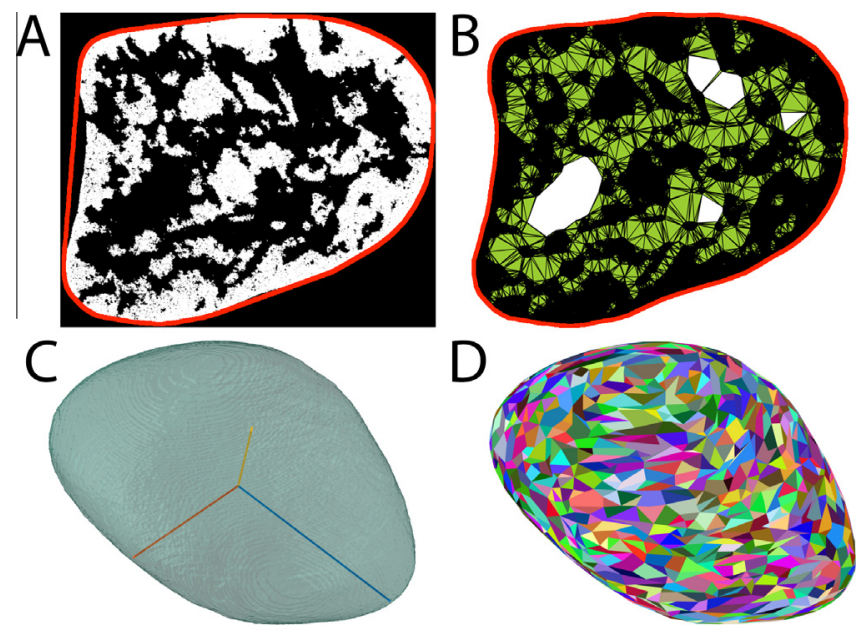

Fig. 3. Nuclear envelope determination. (A) Segmented heterochromatin domains on which the calculated convex hull (plotted in red) represents imperfectly the nuclear envelope due to concavity and disconnections. (B) Nuclear boundary determinated using $\alpha$-shapes represents more accurately the nuclear envelope. (C) Direction and length of the principal axes as determined using principal component analysis on a point cloud of all voxels inside the nuclear envelope. (D) Surface area calculated as the total area of all triangles in the surface mesh representation of the nuclear envelope. method is best suited to discriminate highly contrasted image areas while more subtle contrast differences, such as found between heterochromatin and nucleolus, will need to apply local methods.

\subsection{Size and shape of the nucleus}

The thresholding operation results in a set of connected heterochromatin regions which gives the overall shape of the nucleus (Fig. 3A) even though the boundary of the nucleus, delineated by the nuclear envelope, is not yet readily accessible. We thus need to delineate the nuclear envelope in order to determine the extent of the nucleus and calculate some statistics from the 3-D nuclear shape.

The convex hull method (Andrew, 1979), often used to determine the boundaries of a set of points, was found inappropriate because of the existence of concave segments on the nuclear envelope (Red line in Fig. 3A). We therefore used $\alpha$-shapes (Van Kreveld et al., 2011) to determine the nuclear envelope from the set of segmented heterochromatin domains. The radius $\alpha$ of the circles used to model the boundary of the segmented domains sets the level of details of the final shape. However it should be chosen large enough to ensure that the circle can bridge heterochromatin domains on either sides of a nuclear pore complex. The calculated boundary is thus continuous and represents well the nuclear envelope (Red line in Fig. 3B).

From the calculated 3-D nuclear envelope (Fig. 3C) a number of morphological parameter can be determined such as the total volume of the nucleus, its surface area (Fig. 3D), and its elongation (Fig. 6). The analysis of the 5 rod photoreceptor nuclei shows that their average volume is of $32.2 \mu \mathrm{m}^{3}$ with only small variations of $\pm 2.7 \mu \mathrm{m}^{3}$ mainly due to one outstandingly large nucleus. The mean surface of the nuclear envelope is of $57.2 \mu \mathrm{m}^{2}$ with a similar variation of $8.7 \%$. The elongation ratio, defined here by the ratio between the longest and the shortest orthogonal axes, is of 1.97 but larges variations were found in this parameter ( 0.4 or $20 \%)$ indicating that the shape of the nucleus is less constrained than its volume or the surface of its envelope. No preferential orientation of the longest axis relative to the retina was found in these images.

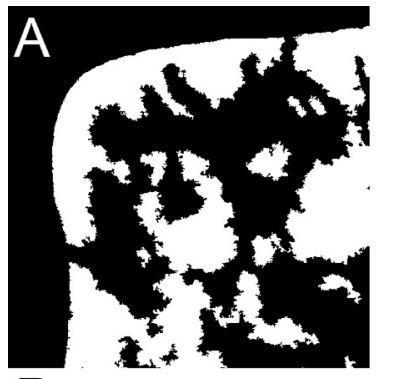

D

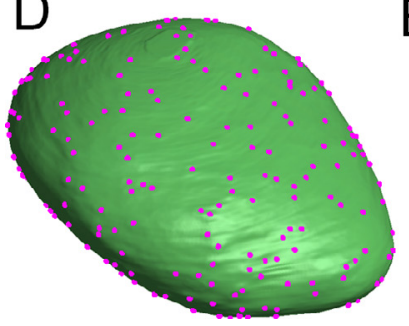

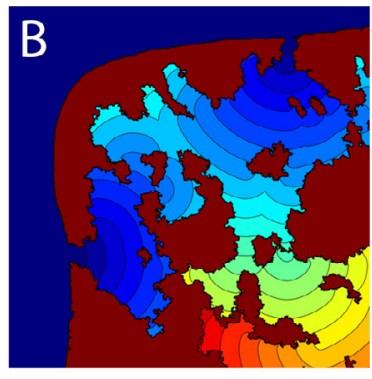

E

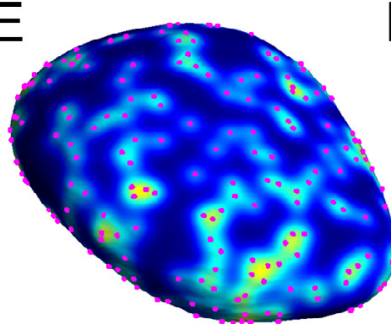

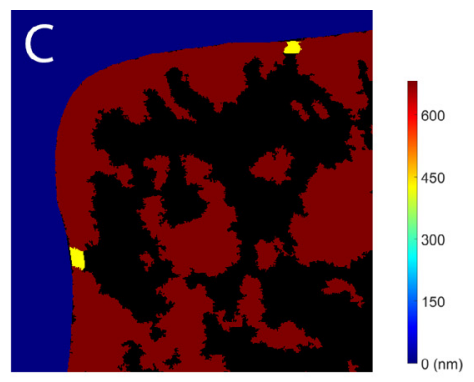

$\mathrm{F}$

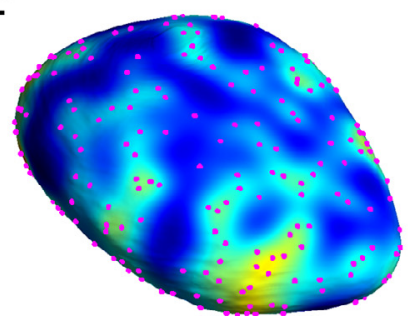

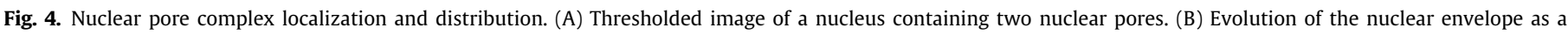

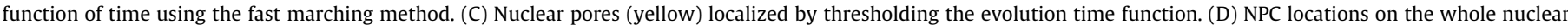

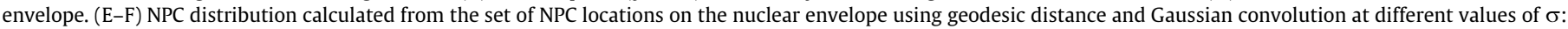
(E) $\sigma=0.15 \mu \mathrm{m}$ and $(\mathrm{F}) \sigma=0.25 \mu \mathrm{m}$

Please cite this article in press as: Hoang, T.V., et al. Automatic segmentation of high pressure frozen and freeze-substituted mouse retina nuclei from FIBSEM tomograms. J. Struct. Biol. (2016), http://dx.doi.org/10.1016/j.jsb.2016.10.005 


\subsection{Nuclear pore complex identification, counts and distribution}

Nuclear pore complexes (NPCs) are important structures that regulate the nuclear-cytoplasmic transport of biological macromolecules. Holes in the nuclear envelope are good indicators for the position of the NPCs. In addition they are always associated with euchromatin domains through which macromolecules can easily be in- and exported. To identify NPCs the most straightforward method is to use a template based approach in which a selected NPC is used as a reference to find similar structures in the full tomogram by 3-D correlation (Hoang et al., 2013). Such an approach is extremely time consuming considering the large size, several tens of gigabites, of FIB-SEM tomograms. A different method was therefore implemented. It is based on the fast marching method (Sethian, 1999) to simulate the evolution of a closed surface (i.e. nuclear envelope) into the euchromatin space over time $T$ through the NPC channels using a specified speed at each location (Fig. 4). NPCs are automatically detected, localized and denoted within a 2-D SEM image after thresholding the evolution time function (Fig. 4B and C). Note that since the speed function $F$ is undefined for the heterochromatin domains in our model, the curved surface cannot evolve into them. Thus, the values of $T$ over those regions are also undefined. The isolated connected regions which remain after thresholding the evolution time reflect the positions of the NPCs. Their exact position is computed as the centroid position of these connected regions (Fig. 4D). The number of NPCs is remarkably constant among the 5 nuclei analyzed with an average value of 348.2 and a standard deviation of only $1.6 \%$ (Fig. 6). Visual inspection and interactive counting of NPCs in one nuclei yielded the same number as our automated protocol. However the identification of NPCs make two assumptions: (i) the NPC has to be associated with a nucleoplamic lumen and NPCs covered by heterochromatin would not be detected by this method. Visual inspection of the nuclear envelope surface did not show such situations. (ii) Two interacting NPCs may be counted as one. This situation is likely to be rare since the NPC apertures were of regular size.

Using geodesic distance calculations combined with a Gaussian convolution (Eq. (3) in materials and methods), we can smoothen the NPC locations to get their distribution (Fig. 4E and F). Even though the distribution depends on the value of the convolution parameter $\sigma$, the color pattern on the nuclear envelope reflects the density of NPCs in each surface area unit. Using this color pattern, we can readily identify a surface region with a higher density of NPCs and, similarly, a surface region depleted of NPC. At this stage of development the distribution appears rather homogeneous and we could not detect any clustering of NPCs.

Interestingly a higher NPC density is generally associated with a large nucleoplasmic cavity in the nucleus (Fig. 5A) while regions devoid of NPCs correspond to large patches of heterochromatin associated with the nuclear membrane (Fig. 5B). Worth noticing is that each NPC is connected to the nucleoplasmic network,
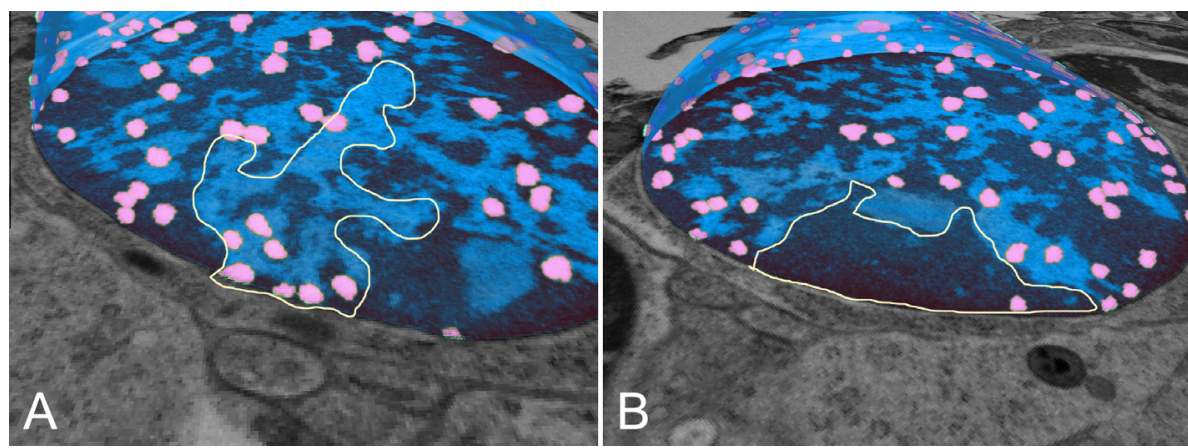

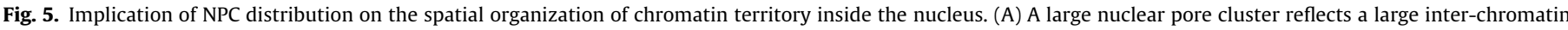

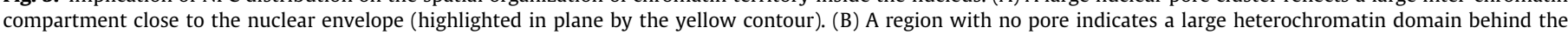
nuclear envelope (highlighted in plane by the yellow contour).
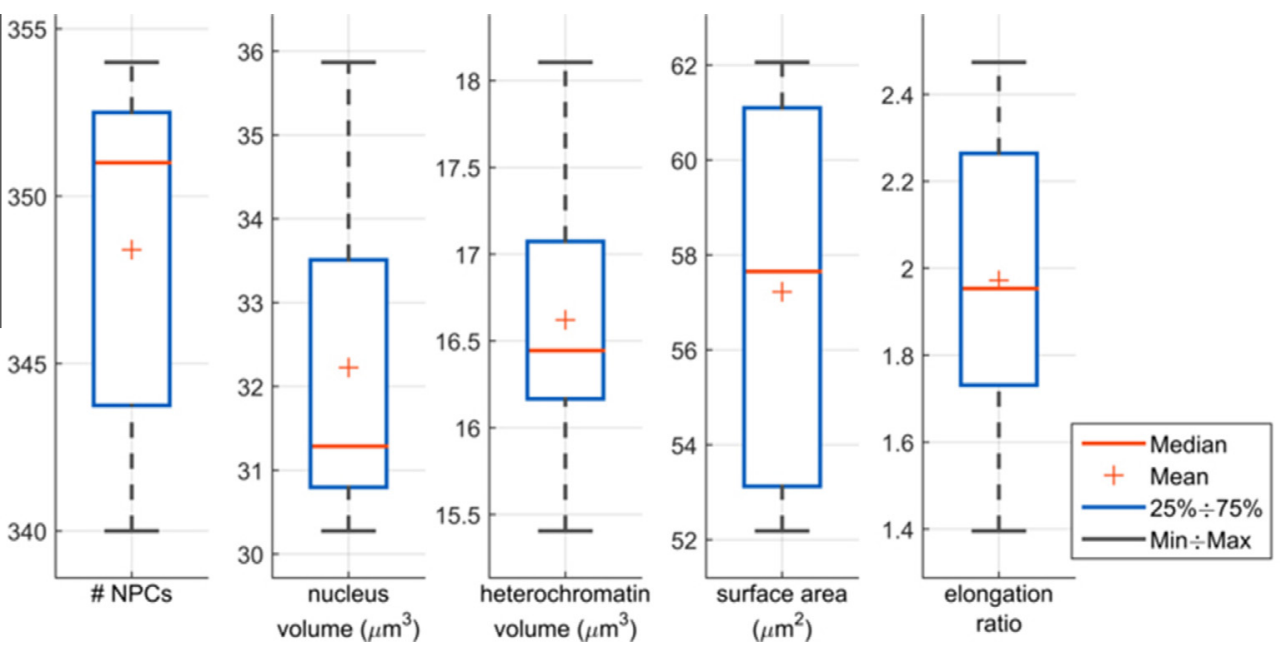

Fig. 6. Box plot of the extracted morphological parameters. The median, mean, minimal and maximal values are shown for each measured parameter. 
sometimes through narrow channels. Consequently, whatever the position of any molecule in the nucleus it can leave the nucleus through any pore.

\section{Discussion}

\subsection{Image pretreatment before segmentation}

The FIB-SEM technology provides 3-D images of cells and tissues with isotropic resolution, thus opening the possibility to extract quantitative information from the collected data. Following the aim of analyzing nuclear images in a semi-automated way, image corrections were found necessary for accurate intensitybased image segmentation and special care was taken to implement methods that reduce noise without loss of spatial resolution and contrast. Global variations in image intensity which could arise from an inhomogeneous illumination or electron detection were corrected by a polynomial surface fitting function which, as compared to a high pass filter, affects less the image contrast and is independent of the spatial frequency cut-off.

The presence of a high level of noise in the SEM images precludes sensible segmentation and entails that peak noise is reduced. Standard low pass filtering smoothens the edges of the hetrochromatin boundaries and results in a less precise segmentation. Edge-preserving CED filters were therefore used in this work. An image intensity histogram was used to select the most appropriate intensity threshold value to discriminate pixels that correspond to heterochromatin domains from those representing nucleoplasmic regions. The intensity histogram clearly showed a bimodal distribution that could be modeled by two Gaussian curves. We however cannot exclude that within the heterochromatin domain, different levels of DNA compaction leading to overlapping intensity distribution can occur.

A hysteresis based threshold was chosen to extend the heterochromatin domains without including sporadic dark pixels present in the nucleoplasm. Despite intensity normalization it was important to determine for each 2-D section the most appropriate set of threshold values since these values were found to drift. However the detection of the corresponding peaks in the intensity histogram allowed a reliable and automated process for segmentation of the heterochromatin domains.

\subsection{Rod nuclei are the smallest among higher eukaryote somatic cell types}

The nuclear volume of 4 days postnatal mouse rod photoreceptors, here estimated at $32.2 \pm 2.7 \mu \mathrm{m}^{3}$, is one of the smallest ever recorded for higher eukaryotes and reflects the high degree of chromatin compaction in these cells. In most eukaryotic cell types the nuclear volume is comprised between 250 and $600 \mu \mathrm{m}^{3}$, with reported exceptions such as drosophila S2 cells or primary human granulosa cells in which the nucleus occupies a volume of $78 \mu \mathrm{m}^{3}$ or $50 \mu^{3}$, respectively (Leuchtenberger et al., 1958; Maul and Deaven, 1977). Assuming a round nuclear shape, we recently estimated at $29 \pm 7 \mu \mathrm{m}^{3}$ the volume of adult mouse rod photoreceptors from cell sections (Kizilyaprak et al., 2010) which is consistent with the reported shrinking of rod nuclei during the late phases or eye development. Nuclear volumes are highly variable from one cell type to the other, during development or metabolic state but our small dataset indicates that for rod photoreceptors at a given time during development the variation in nuclear volume is below $10 \%$ suggesting that nuclear size is tightly controlled. Experiments in fission yeast showed that nuclear size is conditioned by cell size and in particular that the volume of individual nuclei is controlled by the relative amount of cytoplasm surrounding each nucleus while (Neumann and Nurse, 2007). In the very elongated rod cells, nuclei are concentrated in the outer nuclear layer (ONL) while most of the cytoplasmic part of the cell resides in the inner segment (IS) and the phototransduction system partitions in the outer segments. Without breaking rod cell continuum, the ONL is separated from the IS by a cell constriction materialized by the outer limiting membrane (OLM) formed by adherent junctions between photoreceptor and Muller glia cells (West et al., 2008). As a result of this barrier, the rod nucleus is surrounded by a thin cytoplasmic layer which might contribute to nuclear size control. In addition, rod cells express high levels of the linker histone $\mathrm{H} 1$ which might also contribute to form highly compacted chromatin. Small nuclei provide the retina with the possibility to densely pack a large number of cells and thus to improve the efficiency of photon detection and the spatial resolution of the detection.

\subsection{A large part of the genome is packed into heterochromatin}

Heterochromatin was defined as the stain accumulating part of the nucleus which is associated with a high DNA content and reflects highly condensed chromatin domains. The intensity histogram of nuclear pixels showed a bimodal distribution and we could not detect distinct intermediate intensity levels that could be related to distinct levels of chromatin compaction. The hysteresis thresholding method determined a heterochromatin value of $16.6 \mu \mathrm{m}^{3}$, closely matching the value of $17 \mu \mathrm{m}^{3}$ obtained previously for adult rod cells by extrapolating values derived from single thin sections (Kizilyaprak et al., 2010). The apparent higher compaction of the genetic material in adult rods is therefore related to the migration of heterochromatin domains to the center of the nucleus and to a shrinking of the nuclear volume rather than a higher proportion of the genome condensed into heterochromatin. In order to approximate the proportion of the genome condensed into heterochromatin we used the $30 \mathrm{~nm}$ chromatin fiber as a model for highly compacted chromatin (Song et al., 2014). Recent cryo-electron tomography studies indicate that the basic interactions between nucleosomes are conserved in vitro in native compact chromatin (Scheffer et al., 2012). Based on the structure of the 30-nm fiber (Song et al., 2014), the packing density of the left-handed helical 30-nm fiber is of about 6.1-6.4 nucleosomes assembled on a $187 \mathrm{bp}$ DNA per $11 \mathrm{~nm}$ turn. The volume of a highly condensed $30-\mathrm{nm}$ fiber is therefore estimated to $6.387 \mathrm{~nm}^{3}$ for $1 \mathrm{kbp}$ of DNA. The whole mouse genome (2.7 $\left.10^{9} \mathrm{bp}\right)$, compacted into such a fiber would occupy a volume $17.2 \mu \mathrm{m}^{3}$. Despite the unavoidable errors associated to this rough estimation the close match between the estimated value and the measured volume of heterochromatin suggests that at this stage of development, most of the genetic information is highly compacted into heterochromatin.

Heterochromatin segmentation showed that the nucleus is traversed by channels in which the free diffusion of macromolecules is likely to be favored. The distribution of the heterochromatin domains provides a 3-D framework of the diffusion-limited areas of the rod nucleus. This 3-D maze can be used as a structural scaffold to model the diffusion of macromolecules within the nucleus.

\subsection{Highly conserved nuclear pore density}

Most of the quantitative information about nuclear pores found in the literature were obtained from spread freeze-dried and metal-shadowed nuclear envelopes, a process which reveals clearly the nuclear pores and their distribution, but perturbs their relationship with the membrane-associated heterochromatin (Garcia-Segura et al., 1989; Lodin et al., 1978; Maul and Deaven, 1977). The analysis of FIB-SEM images reveals the number and distribution of NPCs in their cellular environment and preserves the 
organization of the membrane associated heterochromatin. The total number of nuclear pores per nucleus was shown to vary massively in the neuronal and neuroglial lineages from 18,451 \pm 2336 (Purkinje cells) to $621 \pm 394$ (granule cells) among neurons, and from $1782 \pm 162$ (protoplasmic astrocytes) to $402 \pm 67$ (oligodendrocytes) among glial cells (Garcia-Segura et al., 1989). Therefore the 4 days post-natal murine rod cells experience the smallest reported number of NPCs (348) with a remarkably low variation within the analyzed nuclei. While NPC number, nuclear size and DNA content can vary substantially in eukaryotic cells the number of pores per surface area appears to be a more conserved feature. For a large variety of cells, and even for the small $3.3 \mu \mathrm{m}^{3} \mathrm{~S}$. cerevisiae nuclei, NPC densities ranging between 8 and $12 \mathrm{NPCs} / \mu \mathrm{m}^{2}$ have been reported. Here we find a slightly smaller value of $6.1 \pm 0.4 \mathrm{NPCs} / \mu \mathrm{m}^{2}$, but comparable values were reported for rat neuronal cells such as granule cells found in the cerebellar cortex $\left(6 \pm 4 \mathrm{NPCs} / \mu \mathrm{m}^{2}\right)$, stellate or basket cells $\left(6 \pm 1 \mathrm{NPCs} / \mu \mathrm{m}^{2}\right)$ and oligodendrocytes ( $6 \pm 1 \mathrm{NPCs} / \mu \mathrm{m}^{2}$ ) (Garcia-Segura et al., 1989).

The distribution of NPC in rod cells was found to be particularly homogeneous and aggregated pores could not be detected as can be found in aging cells or in some pathological processes such as Alzheimer disease (Sheffield et al., 2006) or during Plasmodium falciparum infection (Weiner et al., 2011). This may reflect the young and healthy state of these developing cells. The local NPC density variations are related to the chromatin condensation state and higher NPC density correspond to large nucleoplasmic areas within the underlying nucleus while low NPC density is associated with large domains of heterochromatin associated with the nuclear membrane.

\subsection{Future perspectives}

The methods implemented here for the segmentation of FIB/ SEM images provide new prospects for the automated quantification of several nuclear parameters and complement previous efforts in this field (Rouquette et al., 2009). These new tools will allow us to follow key nuclear features during the late stages of eye development in which murine rod nuclei undergo a drastic reorganization by relocating most of their heterochromatin from the nuclear envelope to the nuclear center (Solovei et al., 2009). Nuclear size and shape, chromatin condensation and location, as well as the number and distribution of NPC are important parameters to characterize nuclear organization not only during development and differentiation, but also during aging and disease. Nuclear invaginations and local ruptures of the nuclear envelop are hallmarks of several cancer cells (Hatch and Hetzer, 2014).

Despite our efforts to reduce dimensional changes during specimen preparation, the volumetric values provided are likely to be different from the volumes found in living cells. The retinas were dissected according to an optimized protocol that preserves the electrophysiological activity of the tissue and were instantly cryo-immobilized by high pressure freezing to prevent chemical fixation which produces tissue shrinkage and changes in physicochemical conditions that could reorganize the cellular ultrastructure (Kizilyaprak et al., 2010). Cryo-fixation is the gentlest way to preserve ultrastructural features even in highly hydrated tissue such as brain (Korogod et al., 2015). The volume increase due to freezing water is partially compensated by the application of high pressure during freezing and the formation of mostly vitreous ice with a density close to liquid water. Therefore it is accepted that high pressure freezing results in negligible dimensional changes. The resin embedding is performed at low temperature $\left(-90^{\circ} \mathrm{C}\right)$ by freeze substitution which prevents any osmotic changes that could result in volumetric changes. Finally, while dimensional changes occur in TEM tomography where electron irradiation results in mass loss and shrinkage, these effects are reduced FIB-
SEM tomography since the X-Y dimensions are set by the underlying block and the $Z$ dimension is set by the milling process. The most noticeable effects are the generation of artefacts from anisotropic milling where streaks appear on the specimen surface along the milling direction (named curtaining) and redisposition of material sputtered from the sample surface but these artefacts do not affect volumetric information.

FIB-SEM images hold more information that still needs to be extracted in an automatized manner and converted into quantitative values usable for correlation with functional aspects. In particular the nucleolus needs to be analyzed in detail since its complex organization and variable texture renders segmentation difficult. Finally small feature with low contrast are visible in the nucleoplasm and any correspond to extended chromatin fibers or transcription-liked perichromatin fibrils for which expert intervention is still needed for structural annotation.

\section{Materials and methods}

\subsection{Specimen preparation}

\subsubsection{Retina dissection}

Four days old C57/BL6 wild-type mice were sacrificed according to institutional ethical guidelines and their eyes were rapidly enucleated and placed in Artificial CerebroSpinal Fluid (ACSF) buffer (126 mM NaCl, $2.5 \mathrm{mM} \mathrm{KCl}, 1.2 \mathrm{mM} \mathrm{MgCl}_{2}, 2.4 \mathrm{mM} \mathrm{CaCl}$, $1.2 \mathrm{mM} \mathrm{NaH} \mathrm{PO}_{2} \mathrm{PO}, 18 \mathrm{mM} \mathrm{NaHCO}_{3}, 11 \mathrm{mM}$ Glucose, PH 7.4, equilibrated with $95 \% \mathrm{O}_{2}$ and $5 \% \mathrm{CO}_{2}$ ) for dissection. The retinas were gently separated from the pigment epithelium and the sclera after removal of the lens, iris and vitreous humor. The dissection was completed in less than 5 min (Wilson et al., 1998).

\subsubsection{High pressure freezing and freeze substitution}

Retinas were prepared as described earlier (Kizilyaprak et al., 2010). For High-pressure freezing (HPF), retinas were punched (Miltex, biopsy punch) to obtain an oriented disk $1.5 \mathrm{~mm}$ in diameter that fits into the specimen carrier. After infiltration for $5 \mathrm{~s}$ in a drop of cryoprotectant containing 10\% dextran (Sigma-Aldrich \# D1662) and 10\% Bovine Serum Albumin (BSA, Sigma-Aldrich \# A4503) v/v in ACSF buffer, each disk was placed onto a $200 \mu \mathrm{m}$ thick flat gold-plated specimen carrier in order to be cryofixed in the HPF machine (EMPACT2, Leica Microsystems).

After HPF, the sample was freeze substituted at $-90{ }^{\circ} \mathrm{C}$ for $80 \mathrm{~h}$ in acetone supplemented with $2 \% \mathrm{OsO}_{4}$ and was subsequently warmed up slowly $\left(1^{\circ} \mathrm{C} / \mathrm{h}\right)$ to $-60^{\circ} \mathrm{C}$ in an automated freeze substitution device (AFS2, Leica Microsystems) (Humbel, 2008). After $8-12 \mathrm{~h}$ the temperature was raised to $-30{ }^{\circ} \mathrm{C}\left(1{ }^{\circ} \mathrm{C} / \mathrm{h}\right)$ and the samples were kept at this temperature for $8-12 \mathrm{~h}$ before being rinsed several times in acetone. The samples were then infiltrated with gradually increasing concentration of Epon (\#21210--LX112 kit, Epon 812 replacement, LAAD, Williston, USA) in acetone $(1: 2$, $1: 1,2: 1$ volume ratio and finally pure Epon) for $2-3 \mathrm{~h}$ while raising the temperature. Addition of pure Epon was performed at room temperature. After polymerization of the resin at $60^{\circ} \mathrm{C}, 100 \mathrm{~nm}$ thin sections were produced using an ultramicrotome (PC PowerTome, RMC Product, Tucson AZ, USA), collected on 200 mesh copper grids and post-stained for $5 \mathrm{~min}$ with $2 \%$ aqueous uranyl acetate, rinsed and incubated for 2 min with lead citrate in order to assess the quality of the cryofixation by the absence of recognizable ice crystal ghosts.

\subsection{FIB-SEM Tomography}

For the FIB-SEM tomography the resin block was glued on an aluminum SEM specimen stub with the EPO-TEK H20S (\#12672- 
20S, EMS, Hatfield, PA, USA) conductive resin in such a way that the osmicated sample overhangs the stub. The stub was placed in the ultramicrotome and the surfaces, respectively normal to the ion beam and normal to the electron beam was trimmed as described previously (Kizilyaprak et al., 2015). The sample was sputtercoated with $30 \mathrm{~nm}$ of platinum using an evaporator (MED-010, Bal-Tec, Balzers, Liechtenstein), then the stub was mounted in the FIB-SEM microscope (Helios NanoLab 650, FEI Company) using the $45^{\circ}$ aluminum SEM-mount (EMS \#75230). The sample was tilted at $7^{\circ}$ to be milled normal to the ion beam and tilted at $45^{\circ}$ to be imaged normal to the electron beam.

The region of interest (ROI) is located by secondary electron (SE) imaging. To avoid re-deposition on the ROI during the milling process, trenches on both sides of the ROI were cut using the ion beam. The milling was done with an accelerating voltage of $30 \mathrm{kV}$, a dwell time of $1 \mu \mathrm{s}$ and a current of $21 \mathrm{nA}$. To reduce the curtaining effect, the surface above the area of interest was polished with the ion beam using an accelerating voltage of $30 \mathrm{kV}, 1 \mu$ s of dwell time and a current of $14 \mathrm{nA}$, with the cleaning cross section mode. Finally, a carbon layer of $1 \mu \mathrm{m}$ was deposited above the ROI using the gas injector system (GIS) and an ion beam of $30 \mathrm{kV}$ at a current of $790 \mathrm{pA}$.

The FIB-SEM tomography of the ROI was performed with the FEI Slice and View G2 softwareTM. The stage was tilted to $7^{\circ}$ in order to mill the surface normal to the ion beam. The working distance was at $4 \mathrm{~mm}$ corresponding to the coincident point of the two beams. Milling was done at $30 \mathrm{kV}$ acceleration voltage, $800 \mathrm{pA}$ current, $1 \mu$ s dwell time and slice thickness of $10 \mathrm{~nm}$. For image acquisition the stage was tilted to $45^{\circ}$ to have the freshly milled blockface normal to the electron beam. The working distance was reduced to $2.2 \mathrm{~mm}$. The cross section images were acquired by detecting backscattered electrons with the through-the-lens detector (BSE-TLD) in immersion mode. The electron beam voltage was set to $2 \mathrm{kV}$, the beam current to $800 \mathrm{pA}$. A dwell time of $3 \mu \mathrm{s}$ was used with the drift correction mode and an average of 8 frames. The image resolution was set to $4096 \times 3536$, with a horizontal field of view of $16 \mu \mathrm{m}$ corresponding to a pixel size of $3.9 \mathrm{~nm}$.

\subsection{Illumination correction}

The SEM images were grey level normalization, stacked with the tif2mrc command of IMOD (Kremer et al., 1996) and then aligned with 1 to 3 passages through tiltxcorr cross-correlation command. After preprocessing we noticed that the images present density variations that are not related to the structure of the sample but are detrimental for subsequent image segmentation. Two types of artifactual density variations have been detected (Fig. 7). The first one is "global" and extends over the whole imaged area. It corresponds to a continuous density variation identified in Fig. 7 by the two near-vertical pink stripes on the left and right sides of the blue stripe in the center. This defect type might arise from the optics of the SEM. The second density variation is "local" and restricted to each nucleus. In this cases, the intensity of the heterochromatin regions of each nucleus changes gradually from its envelope to its center. This effect is likely to originate from a change in chromatin compaction level (Récamier et al., 2014) or a non-uniform staining of the chromatin during sample preparation. The coexistence of these two effects requires that the correction algorithm works on individual nuclei, instead of the whole SEM image, in order to correct them simultaneously.

Due to the lack of reference images and the locale nature of the intensity distortion, we choose to use a single-image retrospective method (Babaloukas et al., 2011) to correct the illumination of each nucleus individually based on polynomial surface fitting (Russ, 2011). Polynomials are known to be a good model to estimate the gradual variations in brightness. In our case, we use quartic polynomials to represent the intensity values of the heterochromatin regions as

$$
\begin{aligned}
f(x, y)= & \left(a_{4} x^{4}+a_{3} x^{3}+a_{2} x^{2}+a_{1} x+a_{0}\right)\left(b_{4} y^{4}+b_{3} y^{3}+b_{2} y^{2}\right. \\
& \left.+b_{1} y+b_{0}\right)
\end{aligned}
$$

where $a_{i}, b_{j}$ are the polynomial coefficients and $(x, y)$ are spatial coordinates. The values of $a_{i}, b_{j}$ are determined by least square fitting using the Levenberg-Marquardt algorithm (Marquardt, 1963). The corrected image $J$ is recovered from the input image $I$ by

$J(x, y)=I(x, y)-f(x, y)+\operatorname{mean}_{(x, y)} f(x, y)$

\subsection{Edge-preserving and edge-smoothing filtering}

SEM images of biological samples are usually noisy because of the low signal to noise ratio and the raster nature of the scanning combined with the use of scan coils. A linear feature such as a membrane or a heterochromatin boundary often appears zigzagged. Thus, we need sophisticated filtering techniques to facilitate the visualization and interpretation of the information contained in these images. Among many filtering techniques that are available in the literature, we selected methods that help alleviate the effect of noise in the subsequent processing steps.

Our purpose is to smooth the SEM images and at the same time preserve the sharp boundaries and the roughness of the hete-

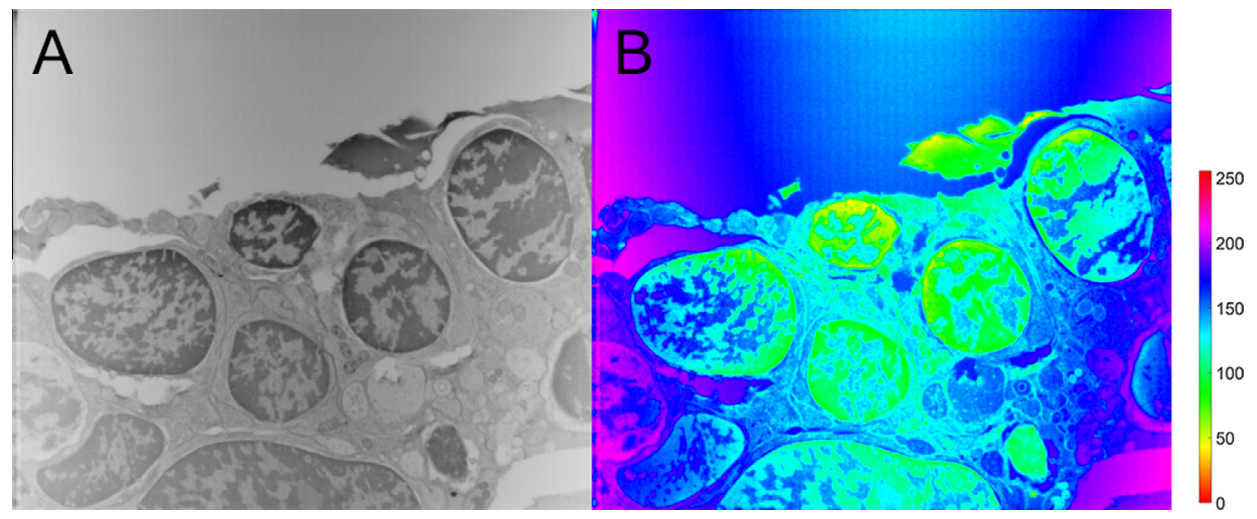

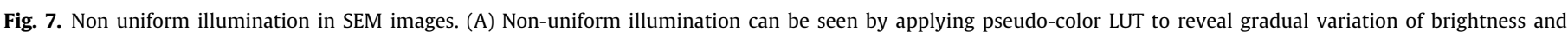

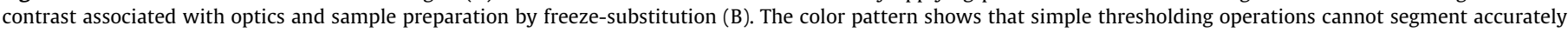
the heterochromatin regions. 
rochromatin edge. We employ the coherence-enhancing diffusion (CED) algorithm (Weickert, 1999) since it has been successfully used to denoise tomography images (Frangakis and Hegerl, 2001). CED is an adaptive Gaussian filtering technique since, for each pixel location, an anisotropic Gaussian function is computed from the structure tensor at that pixel location (Barash, 2002). Note that the edge-preserving characteristics of CED also results in a rough nuclear envelope, which hinders its segmentation and representation. To adapt a filtering method for the detection and representation of the nuclear envelope, we used the block matching 3-D filtering (BM3D) algorithm (Dabov et al., 2007) which smoothes more efficiently the nuclear envelope. BM3D works by exploiting the fact that an image has a locally sparse representation in wavelet transform domain. This sparsity is enhanced by grouping similar 2-D image patches (e.g. blocks) into 3-D data and then applying 3-D collaborative shrinkage-based filtering. The combination of CED and BM3D will facilitate the segmentation of both condensed chromatin domains (by CED) and the nuclear envelop (by BM3D).

\subsection{Hysteresis thresholding}

From the procedure used to stain the nucleus, we know that nucleic acid-rich regions of the nuclei such as heterochromatin areas will accumulate stain and appear dark while euchromatin or nucleoplasmic regions will appear light. We thus use the pixel intensity, after illumination correction and filtering, to partition a nucleus into multiple segments (i.e. sets of pixels) to delineate the boundary between heterochromatin and euchromatin regions. Since this boundary is not smooth and has fractal dimensions (Woodcock and Ghosh, 2010), we choose to use histogram-based methods, instead of contour-based ones (Chan et al., 2001), in order to preserve the roughness of these boundaries.

The darkest pixels correspond most likely to heterochromatin regions which contain tightly packed DNA, while bright pixels correspond to regions with low nucleic acid concentrations such as euchromatin or nucleoplasmic domains. Pixels of intermediate intensity can either belong to heterochromatin or euchromatin regions, and a simple thresholding method will not attribute them to the correct chromatin domain. We reasoned that pixels of intermediate intensity corresponding to heterochromatin domains will stay spatially close and be connected to the darkest pixels. Clustering methods such as k-means (Hartigan and Wong, 1979) or Otsu's (Otsu, 1979) give a single threshold value and thus cannot distinguish these weak dark pixels based on their connectivity with the darkest pixels. In this work, we used the hysteresis thresholding technique (Medina-Carnicer et al., 2009) to track pixels of intermediate intensity that are connected to dark pixels and consider them to belong to the same heterochromatin segments. In case they are not connected to dark pixels, we consider them to belong to euchromatin domains.

Thus, we need two threshold values to classify pixels in the nucleus into three groups: dark pixels, intermediate pixels, and bright pixels. For this purpose, we model the histogram of pixels' intensity by a mixture of two Gaussian distributions (Hastie et al., 2009) to determine the two threshold values $T_{1}$ and $T_{2}$. The left-side Gaussian represents the dark pixels belonging to heterochromatin domains. And the right-side Gaussian represents the bright pixels correspond to euchromatin or nucleoplasmic domains. We set $T_{1}$ as the mean of the left-side Gaussian and $T_{2}$ as the horizontal coordinate of the intersection of the two Gaussians (Fig. 5b).

Having segmented the nucleus into heterochromatin and euchromatin/nucleoplasm domains, we can calculate the total heterochromatin volume of within a single nucleus by multiplying the number of heterochromatin pixels by the voxel size.

\subsection{Nuclear envelope determination}

In computational geometry, the convex hull method (Andrew, 1979 ) is often used to determine the boundary of a set of points $X$ in the Euclidean space. This method gives the smallest convex shape that contains $X$. Although the nuclei of most cells are either round or oval, their shape can contain concave segments particularly in certain pathological conditions (Webster et al., 2009).

$\alpha$-shape is a generalization of the convex hull and a subgraph of the Delaunay triangulation (Van Kreveld et al., 2011). It is calculated by removing all empty circles of radius $\alpha$ and then adding straight edges between any two points that the circle touches to avoid curved edges in the final boundary. From this $\alpha$-shape, we calculate the nuclear envelope as the boundary facets of the triangulation (i.e. the single-face edges). Note that the value of $\alpha$ controls the desired level of detail in the final shape. In our case, $\alpha$ is chosen such that the empty circle of radius $\alpha$ can connect two pixels from the two heterochromatin domains on either sides of a nuclear pore.

After obtaining the nuclear envelope, we can calculate a number of morphological parameters such as the total volume of the nucleus, its surface area, and its elongation. Similar to the total volume of heterochromatin domains, the volume of the nucleus is calculated by multiplying the number of voxels inside the nuclear envelope by the voxel size. The surface area is calculated as the total area of all triangles in the surface mesh that represents the nuclear envelope. For elongation, we use principal component analysis to calculate the direction and length of the principal axes of a point cloud of all voxels inside the nuclear envelope. And the elongation is calculated as the fraction between the length of the longest and shortest axes.

\subsection{Nuclear pore localization and distribution}

Having segmented nuclei and determined their envelope, nuclear pore complexes (NPCs) are clearly identified as appear as holes on the surface of the nuclear envelope. In order to analyze their number and distribution the NPCs need to be localized. Previous work (Winey et al., 1997) located the NPCs manually and analyzed their distribution based on an approximate measure of the distance between of NPCs pair. Here we use the fast marching method (Sethian, 1999) to detect NPCs automatically. The principal idea is to allow the nuclear envelope, represented as a closed surface, to evolve inwards to the euchromatin/nucleoplasm space over time with a pre-defined speed at each location. In our model, the speed function $F$ is defined such that the surface can only evolve with a constant speed into the euchromatin space. This surfaceevolution model allows us to measure, for each location $\mathbf{x}$ inside the nucleus, the time $T(\mathbf{x})$ at which the evolving surface reaches $\mathbf{x}$.

Having defined $F$, the evolution time function $T$ can be computationally calculated by solving the Eikonal equation $F(\mathbf{x})|\nabla T(\mathbf{x})|=1$ using the upwind first-order approximation while taking into account the possible anisotropic voxel size. Since NPCs appear as channels between cytoplasm and nucleoplasm, the values of $T$ at NPC locations should be smaller than the values of $T$ at any other location inside the nucleus. Thus, we can determine the locations of NPCs by simply thresholding $T$. The number of NPCs is the number of connected regions after thresholding.

In order to calculate the NPC distribution over the nuclear envelope, we need to be able to compute the distance $d\left(\mathbf{x}_{i}, \mathbf{x}_{j}\right)$ between any pair of points $\mathbf{x}_{i}$ and $\mathbf{x}_{j}$ on the curved and closed surface in the 3-D Cartesian space. Here we use geodesic distance (Peyré, 2009), which is defined as the length of the shortest path between $\mathbf{x}_{i}$ and $\mathbf{x}_{j}$ on the surface, for this purpose. We again use the fast marching method to compute this geodesic distance by 


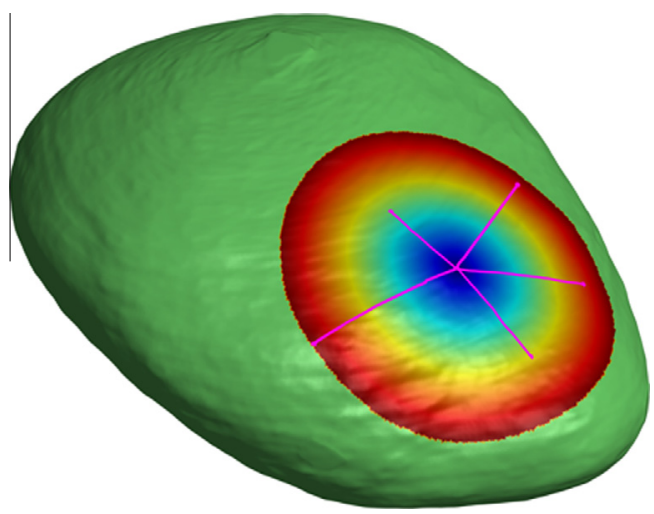

Fig. 8. Geodesic distance calculation between points on the surface mesh of a nuclear envelope. The pseudo-color ring illustrates the distance from the ring's center to other points on the surface mesh. The magenta curves represent the shortest paths, calculated using the gradient descent algorithm, from the ring's center to five other points.

solving the Eikonal equation for surface mesh using Jacobi iterations. Fig. 8 illustrates the geodesic distance from a point to its neighbors on the surface mesh of a nuclear envelope.

For a set of NPCs on the nuclear envelope, we can consider them as a set of Kronecker delta functions $\left\{\delta\left(\mathbf{x}_{i}\right)\right\}_{i}$, where $\mathbf{x}_{i}$ is a NPC location. NPC distribution is then calculated by smoothing these Kronecker delta functions as

$\sum_{i} \mathcal{N}\left(0, \sigma^{2}\right) * \delta\left(\mathbf{x}_{i}\right)=\sum_{i} \frac{1}{\sigma \sqrt{2 \pi}} \exp \left(\frac{d^{2}\left(\mathbf{x}, \mathbf{x}_{i}\right)}{2 \sigma^{2}}\right)$

where $*$ indicates the convolution operation and $\mathcal{N}\left(0, \sigma^{2}\right)$ is a Gaussian function. Note that the geodesic distance $d\left(\mathbf{x}, \mathbf{x}_{i}\right)$ between $\mathbf{x}_{i}$ and any point $\mathbf{x}$ on the nuclear envelope is used when calculating the convolution. In addition, the value of $\sigma$ controls the shape of the Gaussian function and thus affects the final distribution of NPCs. The code and matlab scripts developed for segmentation and NPC counting are available at http://cbi-dev.igbmc.fr/cbi/nucleussegmentation.

\section{Competing interests}

The authors declare no competing financial interests.

\section{Author contributions}

V.T.H performed the image analysis of the FIB/SEM data, C.K. acquired the FIB/SEM data, D.S. prepared the samples for electron microscopy, P.S. and B.M.H. wrote the manuscript. All authors reviewed the manuscript.

\section{Funding}

This work was supported by the Fondation pour la Recherche Médicale, the Institut National de la Santé et de la Recherche Médicale, the Centre National pour la Recherche Scientifique, the Strasbourg University, the Association pour la Recherche sur le Cancer and the Agence Nationale pour la Recherche (CHROMACT), the French Infrastructure for Integrated Structural Biology (FRISBI) [ANR-10-INSB-05-01] and INSTRUCT as part of the European Strategy Forum on Research Infrastructures (ESFRI). By the Faculty of Biology and Medicine of the University of Lausanne and of the Swiss National Science Foundation, R'Equip Grant 316030_128692. TVH was funded by the Labex INRT.

\section{Acknowledgements}

We thank Jonathan Michalon for setting up a competitive computing environment, Dr. Chengge Jiao for suggestion about the optimized sample geometry and Céline Ziegler-Birling for genotyping and taking care of the animals.

\section{References}

Andrew, A.M., 1979. Another efficient algorithm for convex hulls in two dimensions. Inf. Process. Lett. 9, 216-219.

Arbelaez, P., Maire, M., Fowlkes, C., Malik, J., 2011. Contour detection and hierarchical image segmentation. IEEE Trans. Pattern Anal. Mach. Intell. 33, 898-916.

Babaloukas, G., Tentolouris, N., Liatis, S., Sklavounou, A., Perrea, D., 2011. Evaluation of three methods for retrospective correction of vignetting on medical microscopy images utilizing two open source software tools. J. Microsc. 244, 320-324.

Barash, D., 2002. Fundamental relationship between bilateral filtering, adaptive smoothing, and the nonlinear diffusion equation. IEEE Trans. Pattern Anal. Mach. Intell. 24, 844-847.

Bosch, C., Martínez, A., Masachs, N., Teixeira, C.M., Fernaud, I., Ulloa, F., PérezMartínez, E., Lois, C., Comella, J.X., DeFelipe, J., Merchán-Pérez, A., Soriano, E., 2015. FIB/SEM technology and high-throughput 3D reconstruction of dendritic spines and synapses in GFP-labeled adult-generated neurons. Front. Neuroanat. 9,60 .

Bouchet-Marquis, C., Dubochet, J., Fakan, S., 2005. Cryoelectron microscopy of vitrified sections: a new challenge for the analysis of functional nuclear architecture. Histochem. Cell Biol. 125, 43-51.

Bumbarger, D.J., Riebesell, M., Rödelsperger, C., Sommer, R.J., 2013. System-wide rewiring underlies behavioral differences in predatory and bacterial-feeding nematodes. Cell 152, 109-119.

Chan, T.F., Vese, L., 2001. Active contours without edges. IEEE Trans. Image Process $10,266-277$.

Couprie, C., Grady, L., Najman, L., Talbot, H., 2011. Power watershed: a unifying graph-based optimization framework. IEEE Trans. Pattern Anal. Mach. Intell. 33, 1384-1399.

Cretoiu, D., Gherghiceanu, M., Hummel, E., Zimmermann, H., Simionescu, O., Popescu, L.M., 2015. FIB-SEM tomography of human skin telocytes and their extracellular vesicles. J. Cell Mol. Med. 19, 714-722.

Dabov, K., Foi, A., Katkovnik, V., Egiazarian, K., 2007. Image denoising by sparse 3-D transform-domain collaborative filtering. IEEE Trans. Image Process. 16, 2080 2095.

Denk, W., Horstmann, H., 2004. Serial block-face scanning electron microscopy to reconstruct three-dimensional tissue nanostructure. PLoS Biol. 2 (11), e329.

De Winter, D.A.M., Schneijdenberg, C.T.W.M., Lebbink, M.N., Lich, B., Verkleij, A.J., Drury, M.R., Humbel, B.M., 2009. Tomography of insulating biological and geological materials using focused ion beam (FIB) sectioning and low-kV BSE imaging. J. Microsc. 233, 372-383.

Frangakis, A.S., Hegerl, R., 2001. Noise reduction in electron tomographic reconstructions using nonlinear anisotropic diffusion. J. Struct. Biol. 135, 239250 .

Friedrich, R.W., Genoud, C., Wanner, A.A., 2013. Analyzing the structure and function of neuronal circuits in zebrafish. Front. Neural Circuits 7, 71.

Garcia-Segura, L.M., Lafarga, M., Berciano, M.T., Hernandez, P., Andres, M.A., 1989. Distribution of nuclear pores and chromatin organization in neurons and glial cells of the rat cerebellar cortex. J. Comp. Neurol. 290, 440-450.

Gonzalez, R.C., Woods, R.E., 2007. Digital Image Processing. Prentice Hall, Upper Saddle River, N.J..

Hartigan, J.A., Wong, M.A., 1979. Algorithm AS 136: A k-means clustering algorithm. J. R. Stat. Soc. Ser. C Appl. Stat. 28, 100-108.

Hastie, T.J., Tibshirani, R.J., Friedman, J.H., 2009. The Elements of Statistical Learning: Data Mining, Inference, and Prediction. Springer.

Hatch, E., Hetzer, M., 2014. Breaching the nuclear envelope in development and disease. J. Cell Biol. 205, 133-141.

Hekking, L.H.P., Lebbink, M.N., De Winter, D.M., Schneijdenberg, C.T.W.M., Brand, C. M., Humbel, B.M., Verkleij, A.J., Post, J.A., 2009. Focused ion beam-scanning electron microscope: exploring large volumes of atherosclerotic tissue. J. Microsc. 235, 336-347.

Heymann, J.A.W., Hayles, M., Gestmann, I., Giannuzzi, L.A., Lich, B., Subramaniam, S., 2006. Site-specific 3D imaging of cells and tissues with a dual beam microscope. J. Struct. Biol. 155, 63-73.

Heymann, J.A.W., Shi, D., Kim, S., Bliss, D., Milne, J.L.S., Subramaniam, S., 2009. 3D imaging of mammalian cells with ion-abrasion scanning electron microscopy. J. Struct. Biol. 166, 1-7.

Hoang, T.V., Cavin, X., Ritchie, D.W., 2013. GEMfitter: A highly parallel FFT-based 3D density fitting tool with GPU texture memory acceleration. J. Struct. Biol. 184, $348-354$.

Hoppe, W., Grill, B., 1977. Prospects of three-dimensional high resolution electron microscopy of non-periodic structures. Ultramicroscopy 2, 153-168.

Horowitz-Scherer, R.A., Woodcock, C.L., 2005. Organization of interphase chromatin. Chromosoma 115, 1-14. 
Humbel, B.M., 2008. Freeze-substitution. In: Cavalier, A., Spehner, D., Humbel, B.M. (Eds.), Handbook of Cryo-Preparation Methods for Electron Microscopy, Methods in Visualization. CRC Press, pp. 319-341.

Humbel, B.M., Marti, T., Müller, M., 1983. Improved structural preservation by combining freeze substitution and low temperature embedding. Beitr. Elektronenmikrosk. Direktabb Oberfl 16, 585-594.

Humbel, B.M., Schwarz, H., 1989. Freeze-substitution for immunochemistry. In: Verkleij, A., Leunissen, J. (Eds.), Immuno-Gold Labeling in Cell Biology. CRC Press, pp. 115-134.

Jones, C., Liu, T., Cohan, N.W., Ellisman, M., Tasdizen, T., 2015. Efficient semiautomatic 3D segmentation for neuron tracing in electron microscopy images. J. Neurosci. Methods 246, 13-21.

Kasthuri, N., Hayworth, K., Lichtman, J., Erdman, N., Ackerley, C., 2007. New technique for ultra-thin serial brain section imaging using scanning electron microscopy. Microsc. Microanal. 13, 26-27.

Kizilyaprak, C., Longo, G., Daraspe, J., Humbel, B.M., 2015. Investigation of resins suitable for the preparation of biological sample for 3-D electron microscopy. J. Struct. Biol. 189, 135-146.

Kizilyaprak, C., Spehner, D., Devys, D., Schultz, P., 2010. In vivo chromatin organization of mouse rod photoreceptors correlates with histone modifications. PLoS One 5, e11039.

Knott, G., Marchman, H., Wall, D., Lich, B., 2008. Serial section scanning electron microscopy of adult brain tissue using focused ion beam milling. J. Neurosci. 28, 2959-2964.

Korogod, N., Petersen, C.C., Knott, G.W., 2015. Ultrastructural analysis of adult mouse neocortex comparing aldehyde perfusion with cryo fixation. Elife. eLife, 05793.

Kremer, J.R., Mastronarde, D.N., McIntosh, J.R., 1996. Computer visualization of three-dimensional image data using IMOD. J. Struct. Biol. 116, 71-76.

Leighton, S.B., 1981. SEM images of block faces, cut by a miniature microtome within the SEM - a technical note. Scan. Electron Microsc., 73-76

Leuchtenberger, C., Leuchtenberger, R., Doolin, P.F., Shaffer, P., 1958. A correlated histological, cytological, and cytochemical study of the tracheobronchial tree and lungs of mice exposed to cigarette smoke. I. Bronchitis with atypical epithelial changes in mice exposed to cigarette smoke. Cancer 11, 490-506.

Lodin, Z., Blumajer, J., Mares, V., 1978. Nuclear pole complexes in cells of developing mouse cerebral cortex. Vopr. Biokhim. Mozga 13, 152-157.

Luger, K., Mäder, A.W., Richmond, R.K., Sargent, D.F., Richmond, T.J., 1997. Crystal structure of the nucleosome core particle at 2.8 Å resolution. Nature $389,251-$ 260.

Marquardt, D.W., 1963. An algorithm for least-squares estimation of nonlinear parameters. J. Soc. Ind. Appl. Math. 11, 431-441.

Martinez-Sanchez, A., Garcia, I., Asano, S., Lucic, V., Fernandez, J.-J., 2014. Robust membrane detection based on tensor voting for electron tomography. J. Struct. Biol. 186, 49-61.

Maul, G.G., Deaven, L., 1977. Quantitative determination of nuclear pore complexes in cycling cells with differing DNA content. J. Cell Biol. 73, 748-760.

Medina-Carnicer, R., Madrid-Cuevas, F.J., Carmona-Poyato, A., Muñoz-Salinas, R., 2009. On candidates selection for hysteresis thresholds in edge detection. Pattern Recognit. 42, 1284-1296.

Merchán-Pérez, A., Rodriguez, J.-R., Alonso-Nanclares, L., Schertel, A., Defelipe, J., 2009. Counting synapses using FIB/SEM microscopy: a true revolution for ultrastructural volume reconstruction. Front. Neuroanat. 3, 18.

Moor, H., Bellin, G., Sandri, C., Akert, K., 1980. The influence of high pressure freezing on mammalian nerve tissue. Cell Tissue Res. 209, 201-216.

Narayan, K., Danielson, C.M., Lagarec, K., Lowekamp, B.C., Coffman, P., Laquerre, A., Phaneuf, M.W., Hope, T.J., Subramaniam, S., 2014. Multi-resolution correlative focused ion beam scanning electron microscopy: applications to cell biology. J. Struct. Biol. 185, 278-284.

Neumann, F.R., Nurse, P., 2007. Nuclear size control in fission yeast. J. Cell Biol. 179, 593-600.

Noske, A.B., Costin, A.J., Morgan, G.P., Marsh, B.J., 2008. Expedited approaches to whole cell electron tomography and organelle mark-up in situ in high-pressure frozen pancreatic islets. J. Struct. Biol. 161, 298-313.

Nunez-Iglesias, J., Kennedy, R., Plaza, S.M., Chakraborty, A., Katz, W.T., 2014. Graphbased active learning of agglomeration (GALA): a Python library to segment 2D and 3D neuroimages. Front. Neuroinf. 8, 34.

Otsu, N., 1979. A threshold selection method from gray-level histograms. IEEE Trans. Syst. Man Cybern. 9, 62-66.

Oudet, P., Gross-Bellard, M., Chambon, P., 1975. Electron microscopic and biochemical evidence that chromatin structure is a repeating unit. Cell 4, 281-300.

Peyré, G., 2009. Geodesic methods in computer vision and graphics. Found. Trends Comput. Graph. Vis. 5, 197-397.
Porter, K.R., Claude, A., Fullam, E.F., 1945. A study of tissue culture cells by electron microscopy: methods and preliminary observations. J. Exp. Med. 81 (3), 233246.

Porter, K.R., Blum, J., 1953. A study in microtomy for electron microscopy. Anat. Rec $117,685-709$.

Récamier, V., Izeddin, I., Bosanac, L., Dahan, M., Proux, F., Darzacq, X., 2014. Single cell correlation fractal dimension of chromatin. Nucleus 5, 75-84.

Rouquette, J., Genoud, C., Vazquez-Nin, G.H., Kraus, B., Cremer, T., Fakan, S., 2009. Revealing the high-resolution three-dimensional network of chromatin and interchromatin space: a novel electron-microscopic approach to reconstructing nuclear architecture. Chromosome Res. 17, 801-810.

Russ, J.C., 2011. The Image Processing Handbook. CRC Press.

Scheffer, M.P., Eltsov, M., Bednar, J., Frangakis, A.S., 2012. Nucleosomes stacked with aligned dyad axes are found in native compact chromatin in vitro. J. Struct. Biol. 178, 207-214. Special Issue: Electron Tomography.

Schindelin, J., Arganda-Carreras, I., Frise, E., Kaynig, V., Longair, M., Pietzsch, T., Preibisch, S., Rueden, C., Saalfeld, S., Schmid, B., Tinevez, J.-Y., White, D.J., Hartenstein, V., Eliceiri, K., Tomancak, P., Cardona, A., 2012. Fiji: an open-source platform for biological-image analysis. Nat. Methods 9, 676-682.

Sethian, J.A., 1999. Level Set Methods and Fast Marching Methods: Evolving Interfaces in Computational Geometry, Fluid Mechanics, Computer Vision, and Materials Science. Cambridge University Press.

Sheffield, L.G., Miskiewicz, H.B., Tannenbaum, L.B., Mirra, S.S., 2006. Nuclear pore complex proteins in Alzheimer disease. J. Neuropathol. Exp. Neurol. 65, 45-54.

Solovei, I., Kreysing, M., Lanctôt, C., Kösem, S., Peichl, L., Cremer, T., Guck, J., Joffe, B., 2009. Nuclear architecture of rod photoreceptor cells adapts to vision in mammalian evolution. Cell 137, 356-368.

Sommer, C., Straehle, C., Kothe, U., Hamprecht, F.A., 2011. Ilastik: Interactive learning and segmentation toolkit, In: 2011 IEEE International Symposium on Biomedical Imaging: From Nano to Macro. pp. 230-233.

Song, F., Chen, P., Sun, D., Wang, M., Dong, L., Liang, D., Xu, R.-M., Zhu, P., Li, G., 2014 Cryo-EM study of the chromatin fiber reveals a double helix twisted by tetranucleosomal units. Science 344, 376-380.

Tapia, J.C., Kasthuri, N., Hayworth, K.J., Schalek, R., Lichtman, J.W., Smith, S.J., Buchanan, J., 2012. High-contrast en bloc staining of neuronal tissue for field emission scanning electron microscopy. Nat. Protoc. 7, 193-206.

Thoma, F., Koller, T., Klug, A., 1979. Involvement of histone H1 in the organization of the nucleosome and of the salt-dependent superstructures of chromatin. J. Cell Biol. 83, 403-427.

Van den Bergh, M., Boix, X., Roig, G., Gool, L.V., 2014. SEEDS: superpixels extracted via energy-driven sampling. Int. J. Comput. Vision 111, 298-314.

Van Kreveld, M., Van Lankveld, T., Veltkamp, R.C., 2011. On the shape of a set of points and lines in the plane. In: Computer Graphics Forum. Wiley Online Library, pp. 1553-1562.

Webster, M., Witkin, K.L., Cohen-Fix, O., 2009. Sizing up the nucleus: nuclear shape size and nuclear-envelope assembly. J. Cell Sci. 122, 1477-1486.

Weibel, E.R., Kistler, G.S., Scherle, W.F., 1966. Practical stereological methods for morphometric cytology. J. Cell Biol. 30, 23-38.

Weickert, J., 1999. Coherence-enhancing diffusion filtering. Int. J. Comput. Vision 31 $111-127$.

Wei, D., Jacobs, S., Modla, S., Zhang, S., Young, C.L., Cirino, R., Caplan, J., Czymmek, K. 2012. High-resolution three-dimensional reconstruction of a whole yeast cell using focused-ion beam scanning electron microscopy. Biotechniques 53, 4148.

Weiner, A., Dahan-Pasternak, N., Shimoni, E., Shinder, V., von Huth, P., Elbaum, M., Dzikowski, R., 2011. 3D nuclear architecture reveals coupled cell cycle dynamics of chromatin and nuclear pores in the malaria parasite Plasmodium falciparum. Cell. Microbiol. 13, 967-977.

West, E.L., Pearson, R.A., Tschernutter, M., Sowden, J.C., MacLaren, R.E., Ali, R.R. 2008. Pharmacological disruption of the outer limiting membrane leads to increased retinal integration of transplanted photoreceptor precursors. Exp. Eye Res. 86, 601-611.

White, J.G., Southgate, E., Thomson, J.N., Brenner, S., 1986. The structure of the nervous system of the nematode caenorhabditis elegans. Philos. Trans. R. Soc. Lond. B Biol. Sci. 314, 1-340.

Wilson, M.T., Farmer, M.A., Karwoski, C.J., 1998. Ultrastructure of the frog retina after high-pressure freezing and freeze substitution. J. Microsc. 189, 219-235.

Winey, M., Yarar, D., Giddings, T.H., Mastronarde, D.N., 1997. Nuclear pore complex number and distribution throughout the Saccharomyces cerevisiae cell cycle by three-dimensional reconstruction from electron micrographs of nuclear envelopes. Mol. Biol. Cell 8, 2119-2132.

Woodcock, C.L., Ghosh, R.P., 2010. Chromatin higher-order structure and dynamics. Cold Spring Harb. Perspect. Biol. 2, a000596. 